\title{
Spontaneously formed autofocusing caustics in a confined self-defocusing medium: supplementary
} material

\author{
Michael Karpov, ${ }^{1}$ Thibault Congy, ${ }^{2}$ Yonatan Sivan, ${ }^{3}$ Victor Fleurov, ${ }^{1}$ Nicolas \\ PAVLOFF, ${ }^{2}$ AND SHIMSHON BAR-AD ${ }^{1, *}$
}

\author{
${ }^{1}$ Sackler School of Physics and Astronomy, Tel Aviv University, Tel Aviv 69978, Israel \\ ${ }^{2}$ LPTMS, CNRS and Univ. Paris Sud, 91405 Orsay Cedex, France \\ ${ }^{3}$ Unit of Electro-Optical Engineering, Ben Gurion University, Beer Sheva 84105, Israel \\ *Corresponding author: shimshon@post.tau.ac.il
}

This document provides supplementary information to "Spontaneously formed autofocusing caustics in a confined self-defocusing medium," http://dx.doi.org/10.1364/optica.2.001053. The supplementary material comprises data from additional numerical simulations, to support our claims, for which there is insufficient space in the main body of the article, as well as a high-resolution version of a figure which could not be included in the article due to lack of space. We also explain the translation from physical units to the dimensionless model. (c) 2015 Optical Society of America

http://dx.doi.org/10.1364/optica.2.001053.s001

We first present data from computer simulations that show the effects of linear absorption and different static, repulsive potentials. Figure S1 shows the same data presented in Fig. 2 of the article with better definition. Figure S1(a) is a contour plot of the light intensity in the channel as a function of the radial and $z$ coordinates, Fig. S1(b) is a plot of the intensity as a function of the radial coordinate at the exit of the channel, and Fig. S1(c) shows, in a contour plot similar to Fig. S1(a), the propagation of light in free space, behind the exit plane of the nonlinear channel, and in particular the self-generated, self-accelerating, autofocusing ring. The data presented in Fig. S1 were calculated assuming the linear absorption is negligible (although it is an essential ingredient of the thermal nonlinearity that we discuss). Figure S2 shows, in a similar representation, data obtained from an identical simulation, except for adding a (realistic) absorption coefficient of $0.04 \mathrm{~cm}^{-1}$, and a matched, $z$-dependent, thermally-induced static potential (namely reduced heating as a function of the $\mathrm{z}$ coordinate). It can be seen that the behavior remains essentially the same, except that the ring is weaker and autofocusing is slightly slower. Figure S3 shows data obtained without absorption (as in Fig. S1), but with the semicircular static potential changed to a Gaussian form of similar width and with the same maximum value (at the center of the channel). In this case the ring starts forming earlier, as the beam undergoes self-defocusing, but the reflective boundary is essential for transforming it into an autofocusing wave. The "ringing" which is typical of Airy beams is evident. Self-acceleration is not as obvious as in Fig. S1 and Fig. S2, but can still be observed upon close examination of Fig. S3(c). Finally, Fig. S4 is a high-resolution version of Fig. 7(b) in the article, and shows the semi-classical trajectories and the position of the (time dependent) caustic obtained from Eq. (10) in the article.

We now explain in more detail the translation from physical units to the dimensionless semi-classical model. We first note that the mass and time, which are calculated from the expressions given below Eq. (2) of the article, are both functions of $U_{\mathrm{r}=0}$, the potential at the center of the waveguide. We estimate $U_{\mathrm{r}=0}$ from the rate of expansion of the outgoing beam, i.e. the part which undergoes self-defocusing and is not reflected by the boundary. Figure 3(b) is an image of the fluorescence at the output plane of the channel for a 3.5-Watt input beam and a 27\% duty cycle (note that this is about the same average power as in Fig. 3(a) - the radius of the ring is indeed almost identical); Figure 3(c) is a fluorescence image that shows how the ring in Fig. 3(b) focuses to a point after propagating $\sim 6 \mathrm{~cm}$ in free space - the spot at the center of Fig. 3(c) is the auto-focused ring, while the part which undergoes selfdefocusing and is not reflected by the boundary is seen as a pale halo in Fig. 3(c). The expansion rate of this halo allows us to calculate the radius of curvature of the phase fronts of the defocusing component at the channel exit plane, and this curvature, in turn, allows us to calculate the refractive index contrast in the channel, $\Delta n$. From the expression at the center of the LHS column in page 2 of the article we finally obtain $U_{\mathrm{r}=0}=50 \mathrm{~cm}^{-1}$, the mass $\mathrm{m}$, and the conversion factor from $\mathrm{z}$ to $t$. 

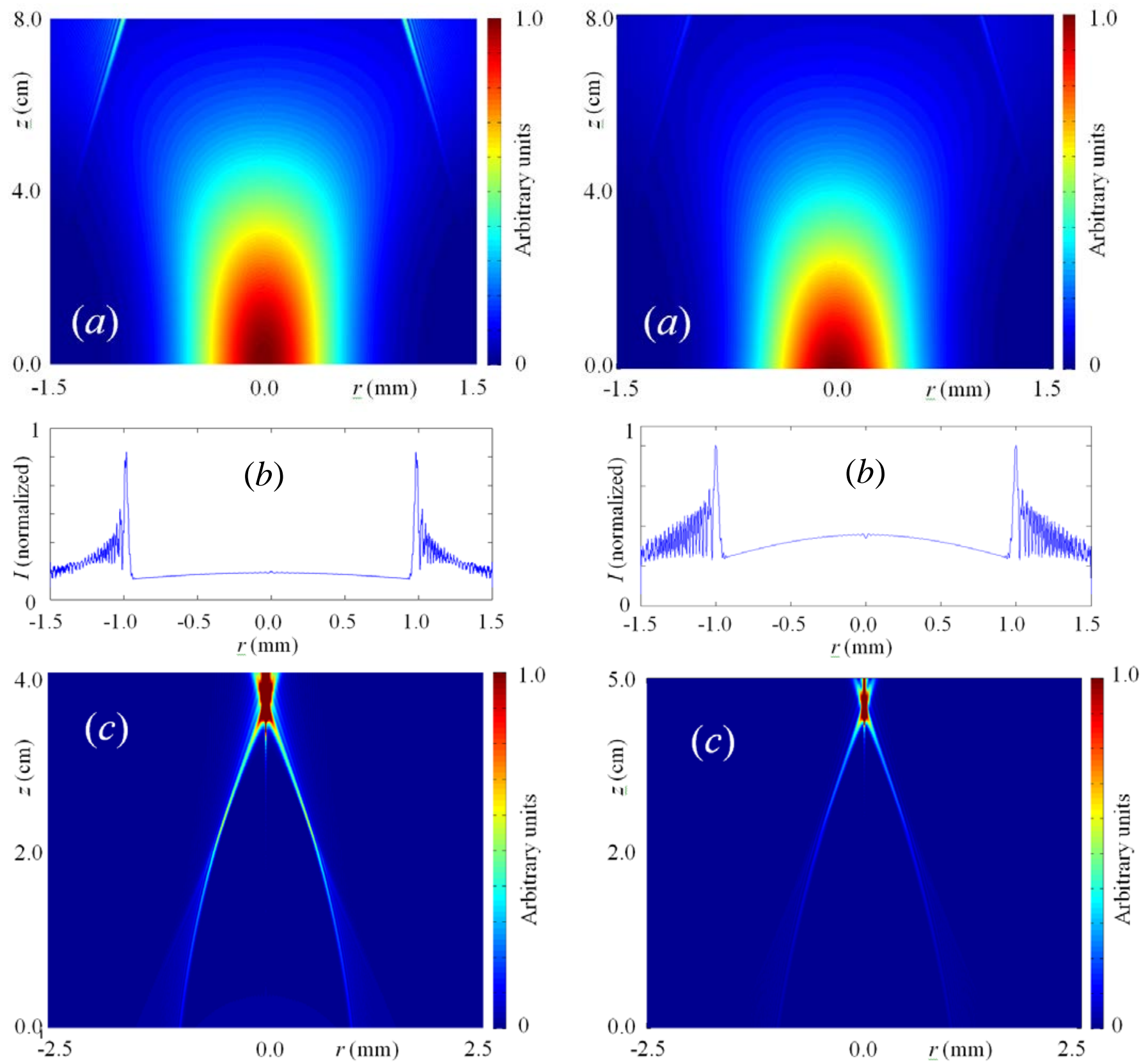

Fig. S1. A numerical simulation of beam propagation through and behind $3 \mathrm{~mm}$ diameter, 8- $\mathrm{cm}$ long channel, with reflective walls, filled with a defocusing nonlinear medium. The input beam is a 1- $\mathrm{mm}$ diameter Gaussian beam, and the other parameters are described in the body of the article and the caption of Fig. 2 therein; (a) is a contour plot of the beam intensity as a function of the radial coordinate and the propagation distance inside the channel; (b) is the intensity profile at the output of the channel; (c) shows self-acceleration and focusing to a point of the output beam as it propagates in free space behind the channel.

Fig. S2. A numerical simulation of beam propagation through and behind the channel, with the same parameters as in the simulation shown in Figure S1, except for the addition of an absorption coefficient of $0.04 \mathrm{~cm}^{-1}$, and a matched, $z$-dependent, thermally-induced static potential (namely reduced heating as a function of the $\mathrm{z}$ coordinate); (a) is a contour plot of the beam intensity as a function of the radial coordinate and the propagation distance inside the channel; (b) is the intensity profile at the output of the channel; (c) shows selfacceleration and focusing to a point of the output beam as it propagates in free space behind the channel. 

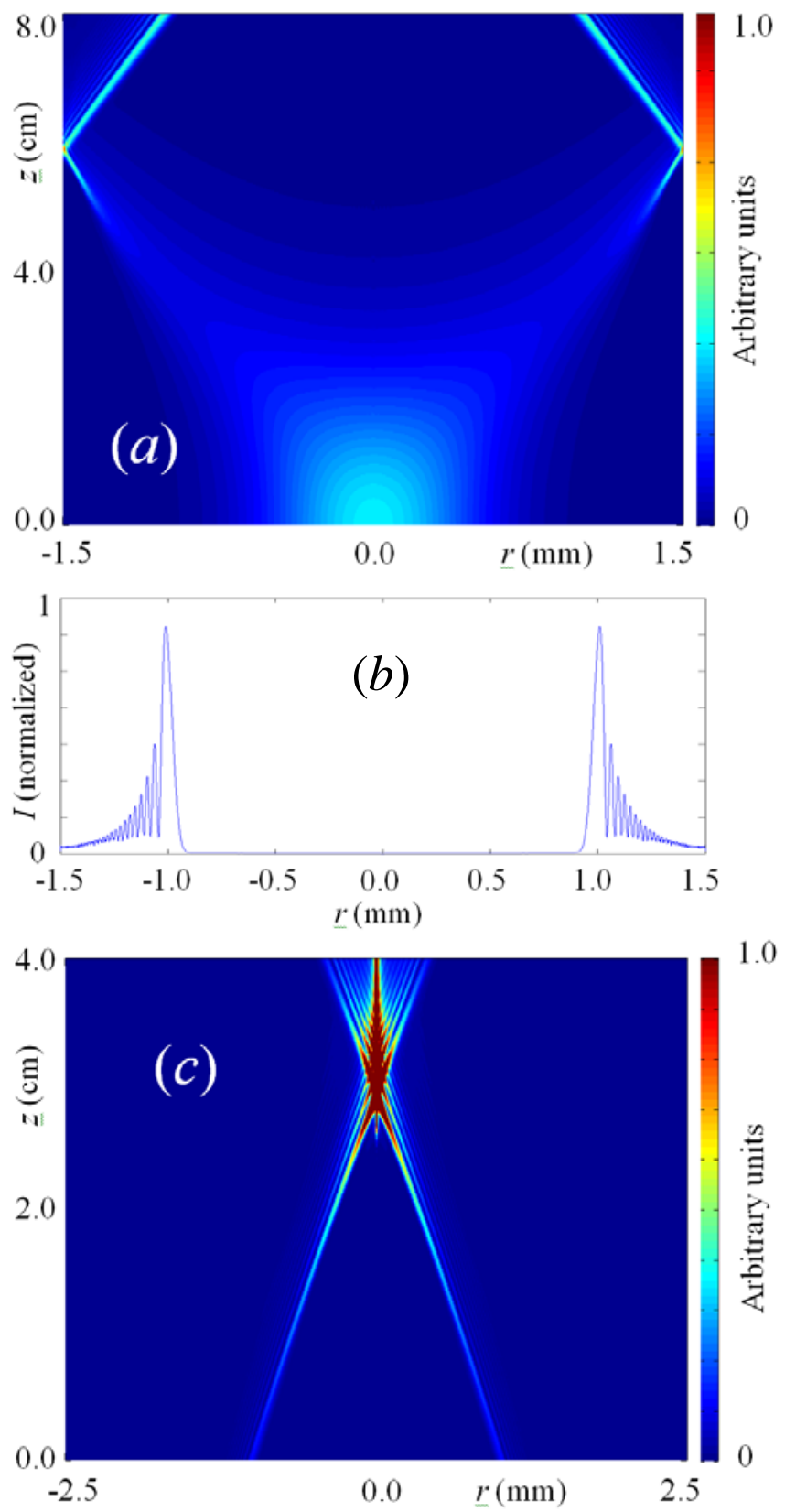

Fig. S3. A numerical simulation of beam propagation through and behind the channel, with the same parameters as in the simulation shown in Figure S1, except for substitution of the semi-circular static potential with a Gaussian form of similar width and with the same maximum value (at the center of the channel); (a) is a contour plot of the beam intensity as a function of the radial coordinate and the propagation distance inside the channel; (b) is the intensity profile at the output of the channel; (c) shows selfacceleration and focusing to a point of the output beam as it propagates in free space behind the channel.

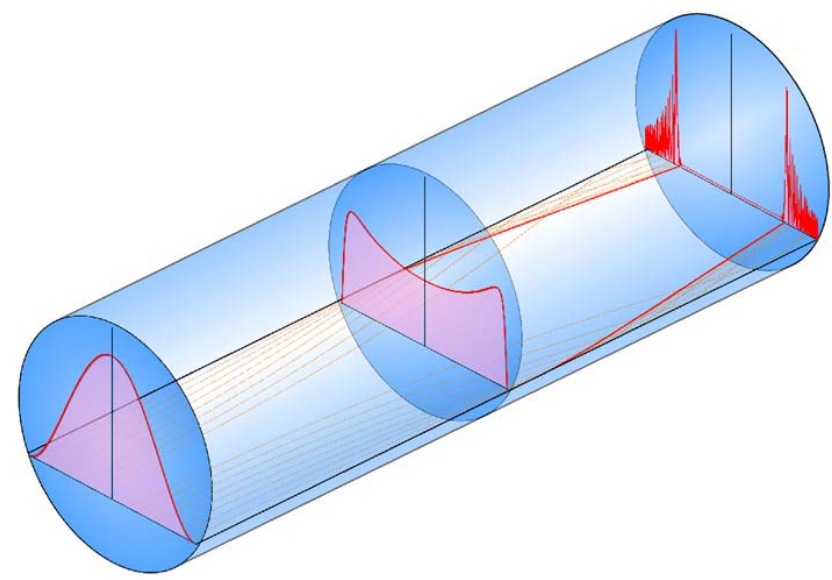

Fig. S4. A sketch of some semi-classical trajectories obtained from Eq. (10) in the article, plotted as orange lines, and the (time dependent) location of the caustic, plotted as a red line; the curves show the evolution of the amplitude of the wave. 